\title{
Études des possibilités diagnostiques sérologiques de l'Onchocercose par double diffusion avec des antigènes hétérologues
}

\author{
par J. PETITHORY, L. BRUMPT et M. BAHNO
}

(Collaboration technique: M. Feillet, A. Ricour et C. VAcher)

Service de biologie médicale. Centre hospitalier, F 95500 Gonesse

\begin{abstract}
Résumé
Les communautés antigéniques entre Nématodes sont utilisées pour le diagnostic sérologique de l'onchocercose. A cet effet, les A.A. étudient la sensibilité et la spécificité de certains antigènes, plus faciles à obtenir que l'extrait homologue: des extraits antigéniques d'Ascaris suum, Neoascaris vitulorum, Parascaris equorum ont donné 83 à $89 \%$ de réactions positives dans l'onchocercose, $20 \%$ dans la Wuchérériose et la dracunculose, et $100 \%$ dans le syndrome de Larva migrans viscérale ou dans quelques cas de poumons éosinophiliques tropicaux. Il n'a pas été observé de fausses réactions chez des témoins non parasités ou des porteurs d'Ascaris n'ayant pas quitté la France.
\end{abstract}

\section{Summary}

Antigenic communities between Nematodes are used for serological diagnosis of onchocercosis. On this surprise, the A.A. Study the sensibility and specificity of antigens, more easy to extract than the homologous antigen: antigenic extracts of Ascaris suum, Neoascaris vitulorum, Parascaris equorum gave 83 to $89 \%$ 
positive reactions in onchocercosis, $20 \%$ in Wuchereriosis and dracunculosis, and $100 \%$ in visceral Larva migrans symptom or in Some cases of tropical eosinophilic lungs. False reactions in non infested people or Ascaris bearers always livine in France have not been observed.

Les communautés antigéniques entre Nématodes sont nombreuses et importantes. Dès 1929, Canning (8) par la méthode de précipitation en tube et au moyen d'immuns sérums de lapins sensibilisés, met en évidence des parentés antigéniques entre $A$. lumbricoides, $P$. equorum, T. canis, A. perspicillum, A. columbae et G. gigas. De son côté, Warren (27-28) démontre expérimentalement, grâce à la réaction de fixation du complément, l'existence de parentés antigéniques entre $W$. bancrofti, $D$. immitis, $T$. spiralis et $L$. carinii. De nombreux autres travaux récents en ont confirmé la réalité.

Ces parentés antigéniques ont été mises à profit dans le diagnostic sérologique de l'onchocercose : elles permettent, en effet, d'utiliser des antigènes plus faciles à obtenir que l'extrait homologue.

Ont ainsi été employés :

- D. immitis (5-9-10-12-21-24-29).

- L. carinii (5-12-24).

- D. vitae (9-10-12).

- Setaria equina (5).

- D. uniformis (10).

- D. medinensis (11-12).

- Loa loa (19-24).

Quant à Ascaris suum, essayé par Bruynoghe (7), sans grand succès d'ailleurs, dans le diagnostic sérologique de la loase, il a donné à Biguet et coll. (4) $76 \%$ de résultats positifs par la méthode d'Ouchterlony dans l'onchocercose. Notre but ici est d'étudier la sensibilité et la spécificité d'un certain nombre d'antigènes de Nématodes pour déterminer les possibilités de leur emploi courant dans le dépistage, si ce n'est le diagnostic, de l'onchocercose.

\section{Matériel et méthodes.}

\section{Onchocercoses.}

Tous les malades sont originaires ou ont séjourné en zone d'endémie onchocerquienne en Afrique: Cameroun, Mali, Sénégal, Tchad, Mauritanie, Côte d'Ivoire, Congo, Gabon, R.C.A., Angola.

Les critères suivants ont été retenus comme base de diagnostic; présence de microfilaires dermiques, nodules, test de Mazzotti positif, isolés ou associés, ce qui permet un diagnostic certain d'onchocercose. 


\section{Méthodes.}

Antigènes : la préparation de l'extrait aqueux a déjà été exposée (18-19-20). Les réactions d'Ouchterlony (16) ont été pratiquées selon une méthode voisine de celle de Hartmann et Toilliez (11) en gel d'agarose à pH 8,2. Les immunoélectrophorèses également en gel d'agarose (6) au même $\mathrm{pH}$.

Les réactions de type substance $\mathrm{C}$-protéine $\mathrm{C}$ ont été éliminées par immersion des lames fraîches dans le citrate de soude à $5 \%$ pendant au moins 4 heures (14). Les traits de précipités de cette nature ne sont donc pas inclus dans nos résultats.

\section{Résultats :}

Ils figurent dans les tableaux suivants :

\section{Tableau I.}

Etude de la sensibilité de divers antigènes pour le diagnostic sérologique de l'onchocercose

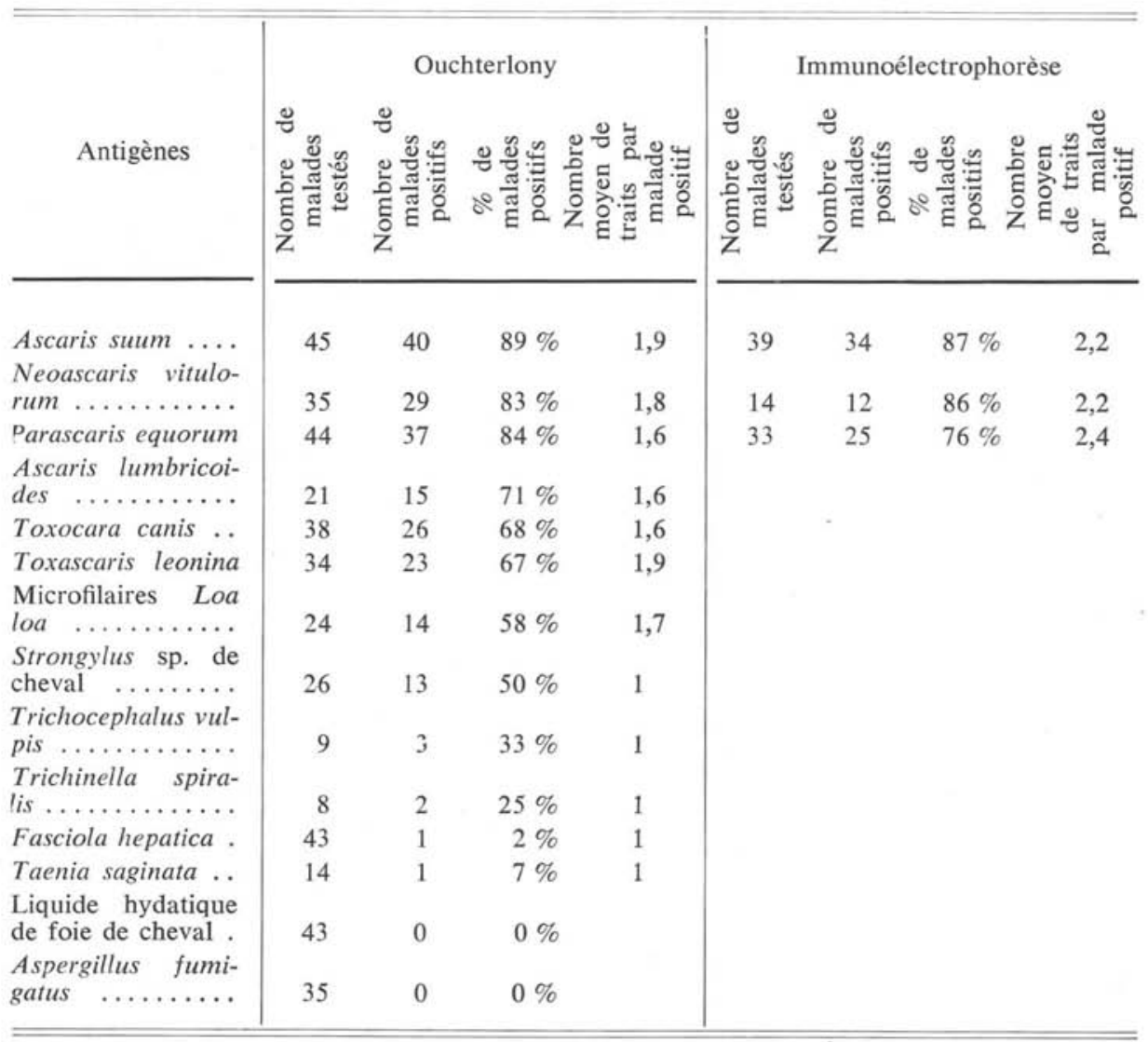


On constate dans le tableau I que trois extraits antigéniques se montrent particulièrement intéressants dans le diagnostic de l'onchocercose: ceux d'Ascaris suum, de Neoascaris vitulorum et de Parascaris equorum qui donnent des résultats positifs dans $83 \%$ à $89 \%$ des cas de malades étudiés.

\section{Tableau II.}

Résultats obtenus avec des antigènes de nématodes dans diverses filarioses

\begin{tabular}{|c|c|c|c|c|c|c|c|c|}
\hline \multirow{2}{*}{$\begin{array}{l}\text { Filarioses } \\
\text { Antigènes }\end{array}$} & \multicolumn{2}{|c|}{ Wuchererioses } & \multicolumn{2}{|c|}{ Dracunculoses } & \multicolumn{2}{|c|}{ Loases } & \multicolumn{2}{|c|}{$\begin{array}{c}\text { Dipetalonema } \\
\text { perstans }\end{array}$} \\
\hline & $\begin{array}{c}\text { Nombre } \\
\text { de cas }\end{array}$ & $\begin{array}{c}\% \text { de cas } \\
\text { positifs }\end{array}$ & $\begin{array}{c}\text { Nombre } \\
\text { de cas }\end{array}$ & $\begin{array}{c}\% \text { de cas } \\
\text { positifs }\end{array}$ & $\begin{array}{c}\text { Nombre } \\
\text { de cas }\end{array}$ & $\begin{array}{c}\% \text { de cas } \\
\text { positifs }\end{array}$ & $\begin{array}{c}\text { Nombre } \\
\text { de cas }\end{array}$ & $\begin{array}{c}\% \text { de cas } \\
\text { positifs }\end{array}$ \\
\hline $\begin{array}{l}A \text {. suum } \\
N . \text { vitulo- }\end{array}$ & 15 & $20 \%$ & 11 & $18 \%$ & 47 & $6 \%$ & 19 & $0 \%$ \\
\hline $\begin{array}{l}\text { rum ... } \\
P . \text { equo- }\end{array}$ & 14 & $14 \%$ & 11 & $27 \%$ & 35 & $6 \%$ & 19 & $0 \%$ \\
\hline $\begin{array}{l}\text { rum } \ldots . . . \\
T . \quad \text { canis }\end{array}$ & $\begin{array}{r}15 \\
6\end{array}$ & $\begin{array}{l}13 \% \\
16 \%\end{array}$ & 11 & $18 \%$ & $\begin{array}{l}45 \\
27\end{array}$ & $\begin{array}{r}4 \% \\
15 \%\end{array}$ & $\begin{array}{r}27 \\
5\end{array}$ & $4 \%$ \\
\hline $\begin{array}{l}\text { T. spira- } \\
\text { lis } \quad \ldots \ldots \text {. }\end{array}$ & & & & & & & 11 & 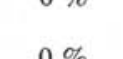 \\
\hline lis $\ldots .$. & 5 & $0 \%$ & & & 16 & $6 \%$ & 11 & $0 \%$ \\
\hline
\end{tabular}

Tableau III.

Réactions à l'égard d'antigènes de nématodes de porteurs d'Ascaris lumbricoïdes intestinaux n'ayant jamais séjournés en zone d'endémie filarienne

\begin{tabular}{|c|c|c|}
\hline Antigènes & Nombre de cas & $\%$ de cas positifs \\
\hline A. suum $\ldots \ldots \ldots \ldots \ldots \ldots \ldots \ldots \ldots$ & 38 & $0 \%$ \\
\hline N. vitulorum $\ldots \ldots \ldots \ldots \ldots \ldots \ldots$ & 13 & $0 \%$ \\
\hline P. equorum $\ldots \ldots \ldots \ldots \ldots \ldots \ldots$ & 24 & $\begin{array}{c}0 \% \\
\text { (1 cas douteux })\end{array}$ \\
\hline T. canis $\ldots \ldots \ldots \ldots \ldots \ldots \ldots \ldots$ & 6 & $0 \%$ \\
\hline
\end{tabular}

Tableau IV.

Parasitoses diverses testées vis-à-vis de l'antigène Ascaris suum

\begin{tabular}{|c|c|c|}
\hline Parasitoses diverses & Nombre-de cas & $\%$ de cas positifs \\
\hline Distomatoses à $F$. hepatica $\ldots \ldots \ldots \ldots \ldots$ & 114 & $14 \%$ \\
\hline Clonorchiase $\ldots \ldots \ldots \ldots \ldots \ldots \ldots \ldots \ldots$ & 7 & $0 \%$ \\
\hline Strongyloïdoses $\ldots \ldots \ldots \ldots \ldots \ldots \ldots \ldots \ldots$ & 32 & $6 \%$ \\
\hline Bilharzioses $\ldots \ldots \ldots \ldots \ldots \ldots \ldots \ldots \ldots$ & 26 & $7 \%$ \\
\hline Syndromes de Larva migrans viscerale ........ & 45 & $98 \%$ \\
\hline
\end{tabular}


Tableau V.

Témoins normaux ou atteints d'affections non parasitaires n'ayant jamais séjourné en zone d'endémie filarienne

\begin{tabular}{|c|c|c|}
\hline Antigènes & Nombre de cas & $\%$ de cas positifs \\
\hline 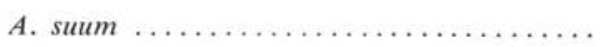 & 248 & $0 \%$ \\
\hline P. equorum $\ldots \ldots \ldots \ldots \ldots \ldots \ldots \ldots \ldots \ldots$ & 248 & $0 \%$ \\
\hline$T$, spiralis $\ldots \ldots \ldots \ldots \ldots \ldots \ldots \ldots$ & 196 & $0 \%$ \\
\hline
\end{tabular}

\section{Discussion :}

$\mathrm{Si}$ nous comparons nos résultats dans le diagnostic sérologique de l'onchocercose avec ceux de différents auteurs ayant utilisé un antigène homologue, nous constatons que les 83 à $89 \%$ des résultats positifs obtenus avec $P$. equorum, $N$. vitulorum et $A$. suum, soutiennent très bien la comparaison avec ceux des auteurs ayant utilisé un antigène extrait d'Onchocerca volvulus : Rodhain et Van den Branden (22), $14 \%$ en fixation du complément, avec il est vrai un extrait alcoolique, Montpellier et Béraud (15), $90 \%$ en fixation du complément avec un extrait aqueux, Van Hoof (26), $86,5 \%$ dans les mêmes conditions, Biguet et coll. (4), 70 à $89 \%$ en Ouchterlony et Ulrich (25), $64 \%$ également en Ouchterlony.

La sensibilité de ces antigènes peut donc être considérée comme équivalente.

La spécificité de ces antigènes, par contre, laisse à désirer, puisque l'on constate avec les trois antigènes précédemment cités environ $20 \%$ de réactions croisées dans les wuchererioses, et les dracunculoses. Dans les syndromes de Larva migrans viscérale. la réaction avec $A$. suum est presque toujours positive. Le taux de $14 \%$ de résultats positifs constatés dans la distomatose à $F$. hepatica est à souligner, ce fait étant donné l'origine géographique habituelle (Europe) de ces malades, pose surtout un problème de diagnostic différentiel entre Larva migrans viscérale et distomatose, problème qui peut d'ailleurs être résolu sérologiquement par la comparaison en Ouchterlony et en immunoélectrophorèse des réactions du sérum du malade avec ces deux antigènes.

Nous avons aussi obtenu des résultats positifs dans trois cas sur quatre de poumon éosinophilique tropical chez des sujets originaires de l'Inde, fait, d'ailleurs, déjà noté par Pacheco (17). Par contre, aucune fausse réaction n'a été notée chez les témoins n'ayant jamais séjourné en zone d'endémie filarienne (Tableau V), même porteurs d'Ascaris lumbricoides (Tableau III) ou de Clonorchis sinensis (Tableau IV).

Enfin, reste le problème des malades atteints de filarioses à $D$. perstans, de loases, de bilharzioses et d'anguilluloses, pour toutes ces affections variées sont retrouvés les deux caractères communs suivants: taux de positivité voisin et faible, de 4 à $7 \%$, malades originaires de pays d'endémie onchocerquienne. Ces données nous conduisent à considérer que ces réactions positives doivent correspondre pro- 
bablement à une onchocercose méconnue. Mais la spécificité des réactions obtenues avec un extrait d'O. volvulus est-elle supérieure?

Montpellier et Béraud (15) obtiennent $50 \%$ de résultats positifs chez des Sénégalais non porteurs de nodules et ont aussi un résultat positif chez un Européen porteur d'oxyures, Van Hoof (26) n'obtient pas de fausse réaction chez un Européen, Biguet et coll. (4) obtiennent $6 \%$ de fausses réactions chez 37 témoins non parasités, deux fausses réactions pour 9 filarioses à $D$. perstans, 1 pour 10 bilharzioses, 4 pour 5 distomatoses, mais pas de fausses réactions non plus pour 18 ascaridioses. On constate donc que cette spécificité est également loin d'être satisfaisante avec un antigène homologue.

Par ailleurs, nous n'avons trouvé aucune corrélation entre la positivité des réactions sérologiques dans l'onchocercose et le taux d'éosinophilie, mais il semble possible, par contre, qu'il existe une relation entre l'âge et le taux des anticorps qui semble diminuer en fonction de celui-ci.

En conclusion, des extraits antigéniques d' $A$. suum, $P$. equorum et $N$. vitulorum nous ont donné de 83 à $89 \%$ de réactions positives dans l'onchocercose, $20 \%$ environ dans la wuchereriose et la dracunculose. $A$. suum a donné aussi $14 \%$ de réactions positives dans la distomatose et près de $100 \%$ dans le syndrome de Larva migrans viscérale ainsi que dans quelques cas de poumons éosinophiliques tropicaux. Par contre, il n'a pas été observé de fausses réactions chez des témoins non parasités ou des ascaridioses n'ayant pas quitté la France.

Les extraits antigéniques d' $A$. suum, $P$. equorum et $N$. vitulorum sont sensiblement équivalents en sensibilité et en spécificité à ceux d'O. volvulus pour le diagnostic sérologique de l'onchocercose.

\section{Bibliographie}

Ambroise Thomas (P.), 1969. - Etude sero-immunologique de dix parasitoses par les techniques d'immuno-fluorescence. Thèse doctorat ès-sciences, Lyon dactyl. 1969.

BaHno (M.), 1972. - Contribution au diagnostic immunologique de l'onchocercose (utilisation d'antigènes hétérologues ascaridiens). Thèse médecine, Paris.

Biguet (J.), D’Haussy (R.), Capron (A.), Tranvan Kv (P.) et Aubry (M.), 1962. Les antigènes de Onchocerca volvulus. I Etude immuno-électrophorétique préliminaire. Bull. soc. path. exot., 55, 845-855.

-, - Aubry (M.) et Rose (F.), 1964. - Etude des anticorps précipitants chez les sujets atteints d'onchocercose. Bull. soc. path. exoct., 57, 1098-1116.

Bozicewich (J.) and Hutter (A. M.), 1944. - Intradermal and serological tests with Dirofilaria immitis antigen in cases of human filariasis. The ann. j. trop. med., 24, 203-208.

Brishammar (S.), Hoerten (S.) and Hofsten (B.), 1961. - Immunological precipitates in agarose gels. Biochim. biophys. acta, 53, 518-521.

BRUYNOGHE (G.), 1939. - Recherches sur les propriétés antigéniques des microfilaires de Dirofilaria immitis. Ann. soc. belge med. trop., 19, 335-353. 
Canning (G. A.), 1929. - Precipitin reactions with various tissues of Ascaris lumbricoïdes and related helminths. The am. $j$. of hyg., 9, 207-226.

Capron (A.), Gentilini (M.) et Vernes (A.), 1968. - Le diagnostic immunologique des filarioses possibilités nouvelles offertes par l'immunoélectrophorèse. Path. biol., $16,1039-1045$.

DUXBURY (R.) and SADUN (E.), 1967. - Soluble antigen fluorescent antibody test (S.A.F.A.) for human filariasis. Exp. parasit., 20, 77-81.

Hartmann (L.) et Toilliez (M.), 1957. - Micro-méthode d'étude en gélose de la réaction antigène anticorps (variante du procédé d'Ouchterlony). Rev. fr. et Clin. biol., 2, 197-199.

Husson (R. A.) et Schweider (J.), 1958. - La réaction de fixation du complément dans le diagnostic des filarioses. Bull. soc. path. exot., 51, 960-967.

KagAN (I.), 1963. - A review of immunologic methods for diagnostic of filariasis. J. parasit., 49, 773-798.

Longbottom (J. L.) and PePYs (J.), 1964. - Pulmonary aspergillosis : diagnostic and immunological significance of antigens and C. substance in Aspergillus fumigatus. J. path. bact., 88, 141-151.

Montpellier (J.) et Béraud (M.), 1921. - La filario réaction chez les sujets atteints de «gale filarienne» (Onchocercose dermique). Bull. soc. dermat. et syph., 28, 344346.

OuCHTERLONY (O.), 1948. - In vitro method for testing the toxin producing capacity of diphteria bacteria. Acta path. microb. scand., 25, 186-191.

Pacheco (G.) and DanRat (T.), 1966. - Indirect hemagglutination with extracts of various helminths in eosinophilic lung (Tropical eosinophilia). Am. j. trop. med. hyg., 15, 355-358.

Petithory (J.), JAy (M.) et Feillet (M.), 1971. - Le diagnostic par immunoélectrophorèse et Ouchterlony de la cysticercose, L'encéphale, 65, 1-12.

-, Brumpt (L.-C.), Jaeger (G.) et SollleuX (M.) avec la collaboration technique de FeilLet (M.), Ricour (A.), Franquert (B.) et Dambrin (A.). - Etude sérologique de la loase en ouchterlony au moyen d'un antigène homologue. Bull. soc. path. exo., 65.

- in Golvan (Y.-J.) et Drouhet (E.), 1972. - Techniques en parasitologie et en mycologie, Paris, Flammarion.

RIDLEY (D.), 1956. - The complement fixation test in filariasis. Trans. roy, soc. trop. med. hyg., 50, 255-257.

Rodhain (J.) et VAN DEN BRANDEN (F.), 1916. - Recherches diverses sur la Filaria (Onchocerca volvulus). Bull. soc. path. exot., 9, 186-198.

Rose (G.), Biguet (J.), Rose (F.) et D'Haussy (R.), 1966. - Application d'une réaction d'hémagglutination au diagnostic de l'onchocercose. Rev. hyg. med. soc., 14, 383392.

Rosseau Baelde (M.) et Janssens (P.-G.), 1961. - Le diagnostic des filarioses humaines à l'aide de la réaction de fixation du complément. Ann. soc. belge med. trop., 41, 329-340.

Ulrich (M.), Pinardi (M. E.) et Convit (J.), 1970. - Immunological reactions in onchocerciasis. Trans. R. Soc. trop. med. hyg., 64, 111-115. 
VAN Hoof (C.), 1934. - Serological reactions in onchocerciasis. Tr. roy. soc. trop. med. hyg., 27, 609-617.

WARren (V.G.), 1947. - Studies on filariasis V Serological relationships between antigenic extracts of four nematodes. T. Am. j. hyg., 45, 299-301.

Warren (K.), Warren (J.) et Hunter (G.), 1946. - Serological relationships between antigenic extracts of $W$. bancrofti and D. immitis. Am. j. hyg., 43, 164-170.

Woodruff (A. W.), Bell (S.), Ridley (D. S.) et Schofield (F. D.), 1958. - Clinical diagnostic and therapeutic aspects of Onchocerciasis. Trans. roy. soc. trop. med., 52, 97-108.

Wiseman et Woodruff, 1971. - Toxocariasis in Africa and Malta. The frequences of infection in host animals and the incidence and distribution in humans as revealed by skin sensitivity tests. Trans. roy. soc. trop. med. hyg., 65, 439-449. 